\title{
Influência das dermatoses \\ na qualidade de vida do portador de diabetes mellitus
}

\author{
The role of dermatosis on diabetes patient's quality of life
}

Adriana N ovaes Rodrigues ${ }^{1}$

Nazaré Pellizzetti Szymaniak 2

Jozias deAndradeSobrinho ${ }^{3}$

${ }^{1}$ Sociedade Frutalense de Ensino Superior. Av Brasília 235, Vila Esperança. 38200-000 Frutal M G. anovaes@ultrasomfrutal.com.br ${ }^{2}$ Centro de Graduação em Enfermagem, U niversidade Federal do Triângulo Mineiro.

${ }^{3}$ Departamento de Cirurgia deCabeça ePescoço e Otorrinolaringologia, Hospital Heliópolis.
Abstract It is known that diabetes mellitus (DM) motives the dermatosis cutaneous because of the proper affection, infections, medicine reactions or the diabetic foot. In this manner, the objective of this study is to work on the hypothesis and identify the influence of the dermatosis in the quality of life of the carrier of DM . It is an analytical, transversal and quantitative study, developed in the Family Health Program (PSF) at Odilom Lacerda, Planura, M inas Gerais State. The sample is composed by $47(100 \%)$ patient carriers of DM, registered in the program called HIPERDIA. Data collection was done by a dermatologist, who applied anamnesis and a chart about theinfluence of the dermatosis in the quality of life during a medical consultation. The analysis of the data was numerical and percentile following the criteria: high frequency ( 80 to $100 \%$ ), average frequency (50 to $79 \%$ ), low frequency ( 1 to $49 \%$ ) and null (for non-existent situations). All the patients of the study (47-100\%) presented dermatosis and demonstrated its influence in quality of life, with averagefrequency for skin drynessand painful sensation in (24-51\%); low frequency, especially the difficulty in the personal hygiene (19-40.3\%) and the interference in the interpersonal relationship with relatives or friends (18-38.2\%).

Key words Dermatosis, Diabetes mellitus, Quality of life
Resumo 0 diabetes mellitus (DM) predispõe às dermatoses associadas à própria afecção, infecções cutâneas, reações medicamentosas ou ao pé diabé tico. Este estudo, analítico, transversal e quantitativo, parteda hipótesedequeas dermatosesinfluenciam a qualidadedevida dosportadores de D M efoi desenvolvido no Programa de Saúde da Família (PSF) Odilom Lacerda, em Planura (M G). A amostra tem 47 (100\%) pacientes portadores de DM e cadastrados no programa HIPERDIA. A coleta de dados foi feita durante a consulta pelo dermatologista, queaplicou a anamnesedirecionada para dermatopatias e qualidade de vida e um formulário para a avaliação subjetiva da influência das dermatoses na qualidade de vida. A análise dos dados foi numérica epercentual, quanto às manifestações provocadas pela dermatose no comprometimento da qualidadedevida, segundo o próprio paciente. Para a mensuração dos resultados, foram estabelecidos os critérios defrequência: alta ( 80 a 100\%), média (50 a $79 \%$ ), baixa ( 1 a $49 \%$ ) enula às situações inexistentes. Todos os pacientes (47-100\%) apresentaram dermatoses e demonstraram que influenciam a sua qualidade de vida, com média frequência no que se refere ao ressecamento e sensação dolorosa na pele (24-51\%) e baixa frequência, especialmentea dificuldade na higienização pessoal (19-40,3\%) ea interferência no relacionamento interpessoal com parentes ou amigos (18-38,2\%).

Palavras-chave Dermatose, Diabetes mellitus, Qualidadedevida 
Introdução

0 diabetemellitus (DM ) éum distúrbio metabólico crônico dos carboidratos, caracterizado por hiperglicemia e glicosúria, resultante da produção ou utilização inadequada da insulina. Aspessoas que preenchem estas condições não formam um grupo homogêneo. O DM éclassificado segundo duas síndromes: o tipo 1 resultaprimariamente da destruição das células beta pancreáticas, com tendência à cetoacidose e o tipo 2 resulta degraus variáveis de resistência à insulina e da deficiência relativa desecreção deinsulina. 0 DM tipo 2 ocorre com frequência maior do que o tipo $1^{1}$.

O DM predispõe a diversas afecções e, notadamente, às doenças cutâneas de natureza variada, porém de etiologia infecciosa em sua maioria. Osportadores deDM são reconhecidos como vulneráveis a uma série de complicações ${ }^{2}$. A pesar da atenção à diabetes, a relação com a qualidade de vida apresenta-se escassa na literatura.

As implicações acarretadas pelo DM envolvem alterações vasculares e neurológicas, as quais muitas vezes contribuem para agravar as condições clínicas vigentes ${ }^{3}$. As complicações do DM são de natureza metabólica e/ou de origem infecciosa, como os processos bacterianos, fúngicose virais $s^{4,5}$

A percepção da qualidade de vida envolve os sentimentos e o comportamento diário do indivíduo. Para Schiper ${ }^{6}$, a qualidade devidaémultifatorial, englobando as áreas física e ocupacional, psicológica, de interação social e da sensação somática. A qualidade de vida relacionada à saúde pode ser mensurada pela avaliação subjetiva do indivíduo no quediz respeito à capacidade de viver plenamente ${ }^{7}$. A atribuição de valor à qualidade de vida é supostamente proporcional à expectativa de vida ${ }^{8}$.

$\mathrm{N}$ a consulta ambulatorial, evidenciam-se dermatoses nos portadores de DM que provavelmente afetam a qualidade de vida desses indivíduos em âmbito geral. N esta pesquisa, parte-se da hipótese de que as dermatoses influenciam a qualidade de vida dos portadores de DM. Portanto, pretende-se obter esta resposta na expressão do próprio paciente, portador de DM, corroborando para a intervenção da equipe interdisciplinar no intuito demelhorar a qualidadede vida destes indivíduos.
Objetivo

0 objetivo deste estudo éi identificar a influência das dermatoses na qualidade de vida do portador de diabetes mellitus.

\section{Método}

Tipo de estudo

Realizou-se um estudo analítico, exploratório, transversal e quantitativo.

Campo de estudo

0 estudo foi desenvolvido no Programa de Saúde da Família (PSF) Odilom Lacerda, no período de julho a agosto de 2005, em Planura, no Triângulo Mineiro, região do Baixo Vale, com aproximadamente 9.000 habitantes 9 . O município conta com duas unidades do PSF, cada um oferecendo cobertura para cerca de 1.200 famílias. O PSF atende a vários grupos de pacientes, incluindo puericultura, gestantes, idosos, hipertensos e diabéticos. 0 grupo de diabetes se reúne semanalmente, realizando-se palestras de orientação, exame clínico e dosagem deglicemia capilar de cada participante diabético.

População do estudo

A amostra deste estudo compõe-se de 47 (100\%) pacientes portadores de DM , atendidos no PSF O dilom Lacerda e todos cadastrados no programanacional de atenção aos pacientes portadores de hipertensão arterial sistêmica e diabetes mellitus, denominado programa HIPERDIA, desenvolvido pelo SistemaÚ nico deSaúde(SUS) e implantado no país desde $2002^{10}$. 0 número total de diabéticos atendidos é de 49 pacientes, sendo que dois pacientes não quiseram participar. Dos 47 pacientes em estudo, doze são insulinodependentes e 35 usam hipoglicemiantes orais.

Aspectos éticos

Após a aprovação deste estudo pelo Comitê deÉtica em Pesquisa (CEP) da Universidade Federal do Triângulo M ineiro (UFTM ), obtiveramseo termo de esclarecimento etermo de consentimento livre e esclarecido (TCLE) para a coleta de dados.

Coleta de dados

A coleta de dados foi realizada durante a consulta médica, pelo pesquisador, queé dermatologista, em sala arejada, de iluminação natural, du- 
rante o período da manhã. Foram aplicados a anamnese direcionada para dermatopatias e qualidade de vida e o formulário para a avaliação subjetiva da influência das dermatosesna qualidade de vida, adaptado do The H ealth Organization Quality of Life(THOQOL) edo Dermatology Life Quality Index (DLQI) ${ }^{11}$, validados no Brasil ${ }^{12,13}$.

O DLQI é um simples e compacto teste de dermatologia, específico e aplicável a pacientes com qualquer doença de pele. M ede quanto afetou um problema de pele a vida do paciente durante os sete dias prévios: consiste em dez artigos, seis dimensões e um score de resumo global, e gamas entre 0 (melhor score) e 30 (pior score). As seis dimensões são: (1) sintomas e sentimentos; (2) atividades diárias; (3) lazer; (4) trabalho e escola; (5) relações pessoais e (6) tratamento. O DLQI está sendo usado em estudos de pacientes com diversas doenças cutâneas ${ }^{14}$. Este questionário mostrou confiança satisfatória evalidez e foi testado para propriedades em vários países, inclusive Brasil ${ }^{12}$.

A cor da pele foi definida como brancos, morenos e negros pelo pesquisador. A queixa principal, em relação à pele, foi questionada diretamente ao paciente. A população foi agrupada por faixa etária. Quando observado o estado civil, foram considerados casados os pacientes que apresentaram companheiro(a) (Tabela 1).

As informações sobre a terapêutica dos pacientes foram obtidas através dos prontuários. 0 exame dermatológico foi realizado com dermatoscópio Episcolp 4500, queéum aparelho de precisão, fabricado por H eine, de origem alemã; trata-se de um equipamento de manipulação manual, com régua milimétrica em seu interior, o que permite a medição das lesões. Não há nenhum tipo de desconforto para o paciente. Não houve necessidade de biópsia cutânea para elucidação de quaisquer lesões cutâneas encontradas.

0 processo decoleta dedadosfoi acompanhado pela enfermeira do PSF, que real izava as anotações necessárias para a real ização desta pesquisa.

Classificação das alterações cutâneas

M inelli et al. ${ }^{15}$ subdividem as alterações cutâneas do DM em quatro grupos:

. Grupo I - lesões fortemente associadas ao DM : necrobiose lipoídica, granuloma anular, doença de Kyrle, escleredema de Buschke, Bullosis Diabeticorum, xantomas, xantomas eruptivos, lipodistrofia congênita generalizada (síndrome de Lawrence-Berardinelli) e pseudoacantose nigricante;

. Grupo II - infecções cutâneas;
Tabela 1. Distribuição dos pacientes portadores de diabete mellitus tipo 1 e 2, por gênero, faixa etária, cor, estado civil, escolaridade, profissão/ocupação. Planura (M G), 2006.

\begin{tabular}{lrr}
\hline \multicolumn{1}{c}{ Aspecto } & $\mathrm{n}$. & \multicolumn{1}{c}{$\%$} \\
\hline Diabetes mellitus & & \\
Tipo 1 & 12 & 25,5 \\
Tipo 2 & 35 & 74,5 \\
Total & 47 & 100,0 \\
Sexo & & \\
M asculino & 20 & 42,6 \\
Feminino & 27 & 57,4 \\
$\quad$ Total & 47 & 100,0 \\
Faixa etária & & \\
Adultos jovens (entre 18 a 29 anos) & 8 & 17,0 \\
Adultos (entre 30 a 59 anos) & 18 & 38,3 \\
Idosos (acima de 60 anos) & 21 & 44,7 \\
Total & 47 & 100,0 \\
Cor & & \\
Branco & 34 & 72,4 \\
Negro & 7 & 14,8 \\
Pardo & 6 & 12,8 \\
Total & 47 & 100,0 \\
Estado civil & & \\
Solteiro & 3 & 6,3 \\
Casado & 22 & 47,0 \\
Separado/divorciado & 8 & 17,0 \\
Viúvo & 14 & 29,7 \\
Total & 47 & 100,0 \\
Escolaridade & & \\
Analfabeto & 5 & 10,6 \\
10 grau & 24 & 51,2 \\
20 grau & 18 & 38,2 \\
Superior & - & - \\
Total & 47 & 100,0 \\
Profissão/ocupação & & \\
Aposentado & 17 & 36,5 \\
Lavrador & 12 & 25,5 \\
Do lar & 9 & 19,1 \\
Lavadeira & 2 & 4,2 \\
Caminhoneiro & 2 & 4,2 \\
Pedreiro & 1 & 2,1 \\
Cabeleireira & 1 & 2,1 \\
Comerciante & 1 & 2,1 \\
Vigia & 1 & 2,1 \\
Policial & 1 & 2,1 \\
Total & 47 & 100,0 \\
\hline & & \\
\hline & &
\end{tabular}

. Grupo III - reações medicamentosas: secundárias ao uso da insulina (edema insulínico, lipoatrofia insulínica ou hipodistrofia lipoatrófica, lipodistrofia hipertrófica ou hipertrofia insulínica, alergia à insulina) ou secundárias ao uso 
dos hipoglicemiantes orais (reações dermatológicas, efeito antabuse) e, finalmente;

- Grupo IV - pé diabético: úlcera neurotrófica plantar, úlcera microangiopática, infecções superficiais eprofundase lesõesisquêmicas por arteriopatia troncular.

Análise de dados

A influência das dermatoses na qualidade de vida de portadores deDM foi analisada numérica e percentualmente quanto à frequência da sua manifestação na vida pessoal, segundo o próprio paciente, em relação a ressecamento ou dor na pele, constrangimento por causa da pele, interferência nas atividades diárias e na maneira de vestir-se, influência no lazer, dificuldade de praticar atividades físicas, interferência no seu trabalho ou atividades escolares, influência no relacionamento interpessoal, problemas relativos à sexualidade devido à pele ou dificuldade na higienização pessoal devido à pele. Para a mensuração da influência das dermatoses na qualidade de vida, foram estabelecidos os seguintes critérios: alta frequência ( 80 a 100\%), médiafrequência ( 50 a $79 \%$ ), baixa frequência ( 1 a $49 \%$ ) e nula às situações inexistentes; critérios prefixados pela validação do Dermatology Life Quality Index (DLQI).

\section{Resultados ediscussão}

Perfil dos pacientes

A população do estudo foi composta por 47 ( $100 \%)$ portadores de DM ecadastrados no Programa de Saúde da Família (PSF) O dilom Lacerda, em Planura, no Triângulo M ineiro. Constatou-se que a maioria é portadora de DM tipo 2 (35-74,5\%), do sexo feminino (27-57,4\%), idoso (21-44,7\%), branco (34-72,4\%), casado (22$47,0 \%)$, escolaridade 10 grau (24-51,2\%), aposentado (17-36,5\%), com renda familiar até dois salários mínimos (23-49\%), demonstrado nas Tabelas 1 e 2.

\section{Queixa principal}

$\mathrm{Na}$ anamnese, a queixa principal dos portadores de DM quanto às lesões cutâneas foi referente às máculas (20-42,5\%), prurido (12$25,5 \%)$, ressecamento (7-14,8\%), além de sentimentos de rejeição relativos à estética, de acordo com a Tabela 3.

Hipoglicemiantes

Neste estudo, dezoito $(38,2 \%)$ pacientes tomavam Diabinese, dez (21,2\%), Daonil, seis,
(12,7\%) Glicoformim e doze ( $25,5 \%)$ eram portadores de DM tipo 1 e dependentes de insulina (DMDI).

Os medicamentos antidiabéticos orais estabelecidos pelo SistemaÚ nico de Saúde(SUS) para fornecimento aos portadores de DM e cadastradosno programa HIPERDIA incluem Glibenclamida 5mg eM etformina $850 \mathrm{mg}$ (ambos via oral), além de insulina humana NPH (injetável), conforme as ações programáticas estratégicas do M inistério da Saúde ${ }^{16}$.

\section{Avaliação dermatológica}

Todos os pacientes deste estudo apresentaram dermatoses (47-100\%). Do ponto de vista dermatológico, constataram-se 146 ocorrências de dermatoses, em média de 3,1 lesões cutâneas por pacientes. De acordo com a Tabela 4, as dermatoses mais frequentes foram a xerose cutânea (29-61,8\%), seguida de dermatofitose, ceratoses actínicas (27-57\%) eelastosecutânea (13-27,7\%),

Tabela 2. Distribuição dos pacientes portadores de diabete mellitus de acordo com a renda familiar. Planura (M G), 2006.

\begin{tabular}{lrr}
\hline \multicolumn{1}{c}{ Renda familiar } & Total $\mathrm{n}$. & \multicolumn{1}{c}{$\%$} \\
\hline Até 1 salário mínimo & 22 & 46,8 \\
Até 2 salários mínimos & 23 & 49,0 \\
Até 3 salários mínimos & 1 & 2,1 \\
Até 4 salários mínimos & 1 & 2,1 \\
Acima de 5 salários mínimos & - & - \\
Total & 47 & 100,0 \\
\hline
\end{tabular}

Tabela 3. Queixa principal quanto à lesão cutânea relatada pelo portador de DM. Planura (M G), 2006.

\begin{tabular}{|c|c|c|c|c|c|c|}
\hline \multirow{2}{*}{$\begin{array}{c}\text { Queixa } \\
\text { principal }\end{array}$} & \multicolumn{2}{|c|}{ Relatado } & \multicolumn{2}{|c|}{ Não relatado } & \multicolumn{2}{|c|}{ Total } \\
\hline & $\mathrm{n}$ & $\%$ & $n$ & $\%$ & $\mathrm{n}$ & $\%$ \\
\hline $\begin{array}{l}\text { "M ancha na } \\
\text { pele" }\end{array}$ & 20 & 42,5 & 27 & 57,5 & 47 & 100,0 \\
\hline $\begin{array}{l}\text { "Coceira na } \\
\text { pele" }\end{array}$ & 12 & 25,5 & 35 & 74,5 & 47 & 100,0 \\
\hline $\begin{array}{l}\text { "Ressecamento } \\
\text { na pele" }\end{array}$ & 7 & 14,8 & 40 & 85,2 & 47 & 100,0 \\
\hline “Pele feia” & 5 & 10,6 & 41 & 89,4 & 47 & 100,0 \\
\hline $\begin{array}{l}\text { "Caroço na } \\
\text { pele" }\end{array}$ & 2 & 4,2 & 45 & 95,8 & 47 & 100,0 \\
\hline “Pele velha” & 1 & 2,1 & 46 & 97,9 & 47 & 100,0 \\
\hline
\end{tabular}


entre outras (23-49,0\%). Algumas dermatopatias diabéticas típicas como a necrobiose lipoídica diabeticorum e o mal perfurante plantar não foram constatadas neste estudo.

Cabe ressaltar que o simples examefísico entre os dedos dos pés contribui para a detecção da xerose cutânea, da dermatofitose e da ceratose actínica, dermatoses de el evada ocorrência entre os portadores do DM , verificadas em $27(57,4 \%)$ dos casos deste estudo.

As dermatofitoses (27-57\%) estão relacionadas às infecções cutâneas, Grupo II na classificação de Minelli ${ }^{15}$, conforme acima descrito. Não foram constatados pacientes com lesões fortemente relacionadas a diabetes, reações medicamentosas ou pé diabético. As lesões cutâneas detectadas neste estudo relacionam-se a alterações provocadas pelo sol e elastose, infecções bacterianas e micóticas.
Dermatoses e qualidade de vida

Os critérios para a mensuração da influência das dermatoses na qualidade de vida de portadores de DM foram de acordo com a frequência das situações referidas pelo pacienteeobedeceram aos critérios estabelecidos: alta frequência (80 a 100\%), média frequência ( 50 a $79 \%$ ), baixa frequência (1 a 49\%) enulas às situações inexistentes.

Este estudo demonstrou não haver situação comum que influencie a qualidade de vida dos portadores de DM, ou seja, com frequência superior a $80 \%$. Ao contrário, houve variação na frequência das situações vivenciadas pelo portador de DM em relação às afecções cutâneas (Tabela 5). Alguns pacientes (10-21,3\%) referiram que as lesões cutâneas incomodam frequentemente o relacionamento interpessoal, enquanto que para outros (6-12,7\%) pouco afeta.

Tabela 4. Classificação das lesões cutâneas em portadores de DM. Planura (M G), 2006.

\begin{tabular}{|c|c|c|c|c|c|c|}
\hline \multirow{2}{*}{ Lesão cutânea } & \multicolumn{2}{|c|}{ Presente } & \multicolumn{2}{|c|}{ Ausente } & \multicolumn{2}{|c|}{ Total } \\
\hline & $n$ & $\%$ & $\mathrm{n}$ & $\%$ & $\mathrm{n}$ & $\%$ \\
\hline Xerose cutânea & 29 & 61,8 & 18 & 38,2 & 47 & 100,0 \\
\hline Dermatofitose & 27 & 57,4 & 20 & 42,6 & 47 & 100,0 \\
\hline Ceratose actínica & 27 & 57,4 & 20 & 42,6 & 47 & 100,0 \\
\hline Elastose cutânea & 13 & 27,7 & 34 & 72,3 & 47 & 100,0 \\
\hline Outras lesões & 23 & 49,0 & 24 & 51,0 & 47 & 100,0 \\
\hline Necrobiose lipoídica & - & - & - & - & 47 & 100,0 \\
\hline Diabeticorum & - & - & - & - & 47 & 100,0 \\
\hline $\begin{array}{l}\text { Mal perfurante plantar } \\
\text { Ausentes }\end{array}$ & - & - & - & - & 47 & 100,0 \\
\hline
\end{tabular}

Tabela 5. Fatores relacionados às dermatoses que influenciam a qualidade de vida do portador de diabetes mellitus. Planura (MG), 2006

\begin{tabular}{|c|c|c|c|c|c|c|c|c|c|c|c|c|}
\hline \multirow{2}{*}{ Frequência/Fatores } & \multicolumn{2}{|c|}{ Muito } & \multicolumn{2}{|c|}{ Ás vezes } & \multicolumn{2}{|c|}{ Pouco } & \multicolumn{2}{|c|}{ Nunca } & \multicolumn{2}{|c|}{ Não pertinente } & \multicolumn{2}{|r|}{ Total } \\
\hline & $\mathrm{n}$ & $\%$ & $\mathrm{n}$ & $\%$ & $\mathrm{n}$ & $\%$ & $\mathrm{n}$ & $\%$ & $n$ & $\%$ & $\mathrm{n}$ & $\%$ \\
\hline Relacionamento & 10 & 21,3 & 2 & 4,2 & 6 & 12,7 & 7 & 14,8 & 22 & 47,0 & 47 & 100,0 \\
\hline Atividades diárias & 9 & 19,1 & 4 & 8,5 & 1 & 2,1 & - & - & 33 & 70,3 & 47 & 100,0 \\
\hline Higienização & 9 & 19,1 & 2 & 4,2 & 8 & 17,0 & - & - & 28 & 59,7 & 47 & 100,0 \\
\hline Ressecamento/dor & 8 & 17,0 & 5 & 10,6 & 11 & 23,4 & 1 & 2,1 & 22 & 46,9 & 47 & 100,0 \\
\hline Constrangimento & 5 & 10,6 & 1 & 2,1 & 7 & 14,8 & - & - & 34 & 72,5 & 47 & 100,0 \\
\hline Lazer & 5 & 10,6 & 3 & 6,3 & 1 & 2,1 & 3 & 6,3 & 35 & 74,7 & 47 & 100,0 \\
\hline Trabalho/escola & 4 & 8,5 & 3 & 6,3 & 2 & 4,2 & 1 & 2,1 & 40 & 85,2 & 47 & 100,0 \\
\hline Vestuário & 2 & 4,2 & 2 & 4,2 & 1 & 2,1 & 5 & 10,6 & 37 & 78,9 & 47 & 100,0 \\
\hline Atividades físicas & 2 & 4,2 & 5 & 10,6 & - & - & - & - & 37 & 78,9 & 47 & 100,0 \\
\hline Sexualidade & 1 & 2,1 & 2 & 4,2 & 3 & 6,3 & - & - & 41 & 87,4 & 47 & 100,0 \\
\hline Total & 55 & & 29 & & 40 & & 22 & & 146 & & & \\
\hline
\end{tabular}


A influência das lesões de pele na qualidade devidade portadores deDM manifesta-secomo:

(24-51\%) ressecamento e sensação dolorosa na pele; soal;

(19-40,3\%) dificuldade na higienização pes-

(18-38,2\%) interferência no relacionamento interpessoal com parentes ou amigos;

(14-29,7\%) interferência nas atividades diárias, como fazer compras, cuidar da casa ou jardim;

(13-27,5\%) constrangimento devido às lesões na pele;

(10-21,1\%) comprometimento das atividades físicas;

(9-19\%) influência no lazer;

(6-12,7\%) interferência no trabalho ou nas atividades escolares, além de problemas relativos à sexualidade devido às lesões de pele e;

(5-10,5\%) influência na maneira devestir-se.

Desse modo, constatou-se que a maior influência das dermatoses na qualidade devida dos portadores de DM refere-se ao ressecamento e sensação dolorosa na pele (24-51\%), seguido de dificuldade na higienização pessoal (19-40,3\%) e interferência no relacionamento interpessoal com parentes ou amigos (18-38,2\%) .

Os dados demonstradosnas Tabelas 5 e 6 confirmam a hipótese de que as dermatoses influenciam a qualidade de vida dos portadores de DM .

\section{Conclusões}

0 estudo mostrou que as dermatoses influenciam a qualidade de vida dos portadores de DM, com média frequência no que se refere ao ressecamento esensação dolorosa na pele (24-51\%) e baixa frequência, especialmente a dificuldade na higienização pessoal (19-40,3\%) .

Interferem também no relacionamento interpessoal com parentes ou amigos (18-8,2\%).

\section{Consideraçõesfinais}

0 impacto de uma doença de pele na qualidade de vida é normalmente mais significante para o paciente, mesmo quando associada a outras patologias ${ }^{17}$. A melhoria da qualidadedevida está sendo considerada como uma meta de tratamento, pois a maioria das patologias crônicas afetam aspectosimportantes da vida de quem as apresenta, sendo que 0 aspecto emocional é o principal. Pacientes com diabetes percebem diferenças significativas nos efeitos de qualidade devida, quando relacionadas às complicações e tratamentos ${ }^{18}$.

0 ressecamento cutâneo é apontado por $K$ ede $^{19}$ como uma patologia frequentemente associada a diabetes, o que torna extremamente importante seu tratamento. Em nosso estudo, observamos que $51 \%$ dos pacientes examinados apresentaram comprometimento da qualidade de vida quando relacionada ao ressecamento e sensação dolorosa.

Tabela 6. Fatores relacionados às dermatoses que influenciam a qualidade de vida do portador de diabetes mellitus. Planura (M G), 2006.

\begin{tabular}{|c|c|c|c|c|c|c|}
\hline \multirow{2}{*}{ Frequência/Fatores } & \multicolumn{2}{|c|}{ Muito + Ás vezes + Pouco } & \multicolumn{2}{|c|}{ Nunca + Não pertinente } & \multicolumn{2}{|c|}{ Total } \\
\hline & $\mathrm{n}$ & $\%$ & $\mathrm{n}$ & $\%$ & $\mathrm{n}$ & $\%$ \\
\hline Ressecamento/dor & 24 & 51,0 & 23 & 49,0 & 47 & 100,0 \\
\hline Higienização & 19 & 40,3 & 28 & 59,7 & 47 & 100,0 \\
\hline Relacionamento & 18 & 38,2 & 29 & 61,8 & 47 & 100,0 \\
\hline Atividades diárias & 14 & 29,7 & 33 & 70,3 & 47 & 100,0 \\
\hline Constrangimento & 13 & 27,5 & 34 & 72,5 & 47 & 100,0 \\
\hline Atividades físicas & 10 & 21,1 & 37 & 78,9 & 47 & 100,0 \\
\hline Lazer & 9 & 19,0 & 38 & 81,0 & 47 & 100,0 \\
\hline Trabalho/escola & 6 & 12,7 & 41 & 87,3 & 47 & 100,0 \\
\hline Sexualidade & 6 & 12,6 & 41 & 87,4 & 47 & 100,0 \\
\hline Vestuário & 5 & 10,5 & 42 & 89,5 & 47 & 100,0 \\
\hline
\end{tabular}


Em relação à higienepessoal, $\mathrm{M}$ ende ${ }^{20}$ relacionou a melhora do quadro deDM ea importância do cuidado com a saúde e integridade dos pés, quando associadaàs condições gerais devidae saúde. Além disso, a intervenção dos grupos de saúde coletiva aumentou a consciência dos pacientes sobre o cuidado com os pés, mostrando os efeitos educacionaisnas complicações da DM ${ }^{21}$. Estas afirmações corroboram com os nossos achados.

Segundo Jóia, Ruiz e Donalisio ${ }^{22}$, um dos parâmetros importantes para a avaliação da qualidade de vida seria a sati sfação, salientando ainda que a satisfação com a vida incluiria aspectos de interação familiar e social, desempenho físico eexercício profissional.

Francioni e Silva ${ }^{23}$ demonstraram que a família assume papel fundamental para a manutenção da qualidade de vida dos pacientes diabé ticos, devido aos limites impostos pela patologia em questão, o que concorda com nosso estudo, no qual $38,2 \%$ dos pacientes apresentaram alterações nas suas relações interpessoais.

H ernández et al.$^{24}$ relataram a necessi dade de uma integração entrea equipedePSF eo pacien- te diabético para melhor êxito no tratamento, permitindo uma continuidade do controle metabólico efísico, favorecen do a melhoria da qualidade de vida destes pacientes.

Assim, estudos que se propõem uma aproximação dos profissionais da saúde com o mundo de quem vive a doença poderão contribuir para o desenvolvimento de uma comunicação mais efetiva a partir da compreensão do que envolve o viver com DM ${ }^{25}$.

Sugere-se, então, quenas consultas ambulatoriais, hospitalização e visita domiciliar haja seguimento interdisciplinar ao portador de DM quanto à influência das dermatoses na qualidade de vida em relação ao ressecamento e dor na pele, dificuldade de higienização, questões relativas ao relacionamento interpessoal, dificuldades nas atividades do dia a dia, enfrentamento do sentimento de constrangimento, superação dos limites na realização de atividades físicas, concretização de lazer, superação das influências negativas no trabalho ou escolares, apoio no âmbito da sexualidade e superação de dificuldades no ato de vestir-se.

\section{Colaboradores}

AN Rodrigues trabalhou na pesquisa e coleta de dados e redação; NP Szymaniak trabalhou na concepção ena redação eJ A Sobrinho trabalhou na redação final do texto. 
Referências

1. Sociedade Brasileira de Diabetes. Diagnóstico e classificação do diabetes melito e tratamento do diabetes melito do tipo 2. Consenso Brasileiro sobre Diabetes 2006. Rio de Janeiro: Diagraphic Editora; 2007.

2. Koivukangas V, Koivukangas V, Annala AP, Salmela $\mathrm{PI}$, Oikarinen $\mathrm{A}$. Delayed restoration of epidermal barrier function after suction blister injury in patients with diabetes mellitus. Diabet. Med. 1999; 16:563-567.

3. Romano G, Moretti G, Di Benedetto A, Giofrè $C$, Di Cesare E, Russo G, Califano L, Cucinota D. Skin lesions in diabetes mellitus: prevalence and clinical correlations. Diabetes Rev Clin Pract. 1998; 39:101-106.

4. Sibbald RG. The skin and diabetes mellitus. Int J Dematol 1984; 23:567-584.

5. Shemer A, Bergman R, Linn S, Kantor Y, FriedmanBirnbaum R. Diabetic dermopathy and internal complications in diabetes mellitus. Int J Dermatol 1998; 37:113-115.

6. Schipper KGP, Baisden WT, Elliott AH. Skin disorders in diabetes. Rev. Clin. Diabetes 1982; 369-379.

7. Sociedade Brasileira de Diabetes. Oliveira JEP. Pre venir é possível. Disponível em: http://www.diabetes. org.br

8. Gonzàlez WBL. Disfunção endotelial no diabetes do tipo 2. Arq. Bras. Endocrinol. M etab. 2002; 46:514519.

9. Instituto Brasileiro de Geografia e Estatística. Ministério do Planejamento. Orçamento e Gestão - Cidades 2007. [site da Internet] [acessado 2007 dez 20]. Disponível em: http://www.ibge.gov.br/cidadesat/ default.php

10. Brasil. M inistério da Saúde. Secretaria de Políticas de Saúde. Departamento de Ações Programáticas Estratégicas. Plano de reorganização da atenção à hipertensão arterial e ao diabetes mellitus: hipertensão arterial e diabete melittus. Brasília: Ministério da Saúde; 2001. p. 68

11. Finlay AY, Khan GK. Dermatology Life Quality Index (DLQI)- a simple practical measure for routine clinical use. Clin Exp Dermatol 1994; 19(3):210-216.

12. Cicconeli RM. Portuguese translation and validation of a generic questionnaire for evaluation of quality of life, "M edical Outcome Study 36-item Short-Form Health Survey (SF-36)" [tese]. São Paulo (SP): U niversidade Federal de São Paulo; 1997.

13. Bradley C, Todd C, Gorton T, Symonds E, Martin A, Plowright R. The development of an individualized questionnaire measure of perceived impact of diabetes on quality of life: the ADDQol. Quality of Life Research 1999; 8:79-91.

14. Coghi S, Bortoletto MC, Sampaio SAP, Andrade Ju-

15. nior HF, Aoki V. A qualidade de vida é muito comprometida em pacientes adultos com dermatite atópica no Brasil, especialmente devido a fatores emocionais. Clinics [periódico na Internet] 2007 [acessado 2007 dez 24];62(3):[cerca de 8 p.]. Disponível em: http://www.scielo.br/scielo.php?script=sci_arttext \& pid=S1807-59322007000300006\&lng=pt\&nrm=iso M inelli L, Nonino AB, Salmazo JC, Neme L, Mar- condes M. Diabetes mellitus e afecções cutâneas. An. Bras. Dermatol 2003; 78:735-747.

16. Brasil. Ministério da Saúde. Portaria no 371/GM de 04 de março de 2002. Cria o Programa Nacional de Assistência Farmacêutica para Hipertensão Arterial e Diabetes Mellitus. Diário Oficial da União 2002; 06 mar.

17. Halioua B, Beumont M G, Lunel F. Quality of life in dermatology. Int J Dermatol 2000; 39(11):801-806.

18. Huang ES, Brown SES, Ewigman BG, Foley EC, M eltzer DO. Patient Perceptions of Quality of Life With Diabetes-Related Complications and Treatments. Diabetes Care 2007; 30(10):2478-2483.

19. Kede M PV, Figueira AL, Porto JA. M anifestações cutâneas no diabetes mellitus. An. bras. dermatol. 1993; 68(1):21-24.

20. M endes M RSSB, Navakoski LER. O cuidado com os pés do senescente: um processo em construção. Texto Contexto Enferm 2000; 9(2 Pt 2):752-763.

21. Sumikawa M, Egawa T, H onda I, Yamamoto Y, Sumikawa $Y$, Kubota M. Effects of foot care intervention including nail drilling combined with topical antifungal application in diabetic patients with onychomycosis. J Dermatol 2007; 34(7):456-464.

22. Joia LC, Ruiz T, Donalisio M R. Condições associadas ao grau de satisfação com a vida entre a população de idosos. Rev. Saude Publica [periódico na Internet] 2007 [acessado $2007 \mathrm{dez} 24$ ];41(1):[cerca de 8 p.]. Disponível em: http://www.scielo.br/scielo. php?script=sci_arttext\&pid=S0034-891020070001 $00018 \& \operatorname{lng}=\mathrm{pt} \AA \mathrm{n} r \mathrm{~m}=\mathrm{iso}$

23. Francioni FF, Silva DGV. O processo de viver saudável de pessoas com diabetes mellitus através de um grupo de convivência. Texto Contexto Enferm [periódico na Internet]. 2007 [acessado 2007 dez 21]; 16(1):[cerca de 7 p.]. Disponível em: http://www. scielo.br/scielo.php?Script=sci_arttext\& pid=S0104$07072007000100013 \&$ Ing $=p t \& n r m=i s o$

24. Quirantes AH, Lopez LG, Curbelo VS, Luna JAM, M achado PL, Quirantes AM. La calidad de la vida del paciente diabético. Rev. Cuba med gen integr [periódico na Internet] 2000 [acessado $24 \mathrm{dez}$ 2007];16(1):[cerca de 7 p.]. Disponível em: http:// scielo.sld.cu/scielo.php?script=sci_arttext $\&$ pid $=$ S0864-21252000000100009\&lng=es\&nrm=iso

25. Silva DM GV. Narrativas do viver com diabetes mellitus: experiências pessoais e culturais. Florianópolis (SC): Programa de Pós-graduação em Enfermagem, UFSC; 2001.

Artigo apresentado em 21/06/2007

Aprovado em 14/12/2007 